\title{
Physical modeling of a microlens array setup for use in computer generated IP
}

\author{
Spyros S. Athineos ${ }^{\mathrm{a}}$, Nicholas P. Sgouros ${ }^{\mathrm{a}}$, Panagiotis G. Papageorgas ${ }^{\mathrm{b}}$, Dimitris E. Maroulis ${ }^{\mathrm{a}}$, \\ Manolis S. Sangriotis ${ }^{\mathrm{a}}$, Nikiforos G. Theofanous ${ }^{\mathrm{a}}$ \\ ${ }^{a}$ Dept. of Informatics \& Telecommunications, Univ. of Athens, Athens, Greece 15784 \\ ${ }^{\mathrm{b}}$ Electronics Dept., Technological Educational Institute of Piraeus, Athens, Greece 12244
}

\begin{abstract}
One of the most promising techniques for visualizing three-dimensional objects is Integral Photography (IP). Two of the most common methods used in Computer Generated IP involve the development of simplified ray tracing algorithms for elementary 3D objects or the realization of pinhole arrays. We present an alternative technique based on the POV-Ray software package for ray tracing to generate synthetic, high quality and photorealistic integral images, by accurately modeling all the optical elements of the capturing setup. Our work constitutes a straightforward approach for translating a computer generated 3D model to an IP image and a robust method to develop modules that can be easily integrated in existing ray-tracers. The proposed technique simulates the procedure of a single stage IP capture for producing orthoscopic IP images, real or virtual. Full control is provided over geometry selection, size and refractive index of the elementary microlenses. Specifically our efforts have been focused on the development of arrays with different geometries (square or hexagonal) that demonstrates the parameterization capabilities of the proposed IP setup. Moreover detailed benchmarking is provided over a variety of sizes and geometries of microlens arrays.
\end{abstract}

Keywords: Integral Photography, Integral Imaging, 3D Displays, Microlens Arrays, Ray-tracing

\section{INTRODUCTION}

Integral Photography (IP) or Integral Imaging (II), devised by Lippmann ${ }^{1}$ in 1908 , is one of the most promising methods for displaying three-dimensional (3D) images since it offers autostereoscopic capabilities together with continuous parallax both horizontally and vertically. At the early stages of IP a typical capturing setup consisted of a microlens array (MLA) placed between the object and a photographic plate or film. Reproduction of IP images captured in this manner was possible in printed form, thus limiting the outcome to static images that lost some of the vividness of the initial scene. Today there is a revitalizing interest in IP due to advances in micro optical manufacturing techniques, the development of high resolution LCDs and CCD's together with increased computational power. These technological advances fulfill the increased demand of IP for high resolutions in order to produce high-quality integral images.

Currently, it is common practice to use computers for the construction and presentation of 3D scenes. Computer Generated Integral Photography (CGIP) ${ }^{2}$, belongs to this general category and aims to the production of Integral Photography images for three-dimensional imaging. A number of software models have been reported ${ }^{3,4}$ that produce integral images mostly by tracing the path of the light rays in a $3 \mathrm{D}$ scene. Variations of these models have used pinhole lenslets ${ }^{3}$ eliminating aberrations, along with simple algorithms of minimal computational requirements, thus providing IP images that were practically rendered in real time. However the main disadvantage of such an approach is a significant degradation of the generated IP images, thus limiting their use in photorealistic applications. Most recently elementary fully aperture lens modeling has been proposed ${ }^{4}$, taking into account lens aberrations and using basic ray-tracing algorithms in order to overcome these limitations. In addition, interpolative shading techniques were used to improve the realism of the integrated image. This method provides integral images of adequate quality but has restrictions in the complexity of the 3D scenes, while the employed ray-tracing algorithms are simplified, compromising quality with computational requirements focused on horizontal parallax IP images. 
The method described in this text, is a computer simulation of a physically implemented single step Integral Imaging capture scheme $e^{5}$, based on the POV-Ray software package ${ }^{6}$, which is used as the ray-tracing engine. The lens array and an imaging lens are modeled as ordinary objects of the 3D scene thus providing great flexibility in the size and geometry of the lens array, supporting various resolutions of the display device.

\section{SINGLE STAGE IP CAPTURING SETUP FOR ORTHOSCOPIC IMAGES}

The single stage IP capturing setup that which is simulated with the POV-Ray ray-tracing software is shown in Fig.1. It corresponds to either a real or a virtual IP setup finally producing an orthoscopic integral image (real or virtual). The imaging lens forms an inverted and demagnified real image with the MLA placed in the image space. The relative distance D of the MLA in regard to the central plane of the image space, determines the IP mode as real or virtual. If the MLA is placed at the end of the image space towards the imaging lens as in Fig. 1 then the pickup integral image is formed at the Pickup Plane at a distance $\mathrm{g}$ from the MLA and the captured integral image is a pseudoscopic virtual image. The elemental images created on the Pickup Plane by the microlens array, are then rotated, using a software method, by 180 degrees, resulting in a real orthoscopic image. If the MLA is placed at the end of the image space towards the camera, the captured integral image is real pseudoscopic and the final outcome after elemental images rotation is a virtual orthoscopic integral image.

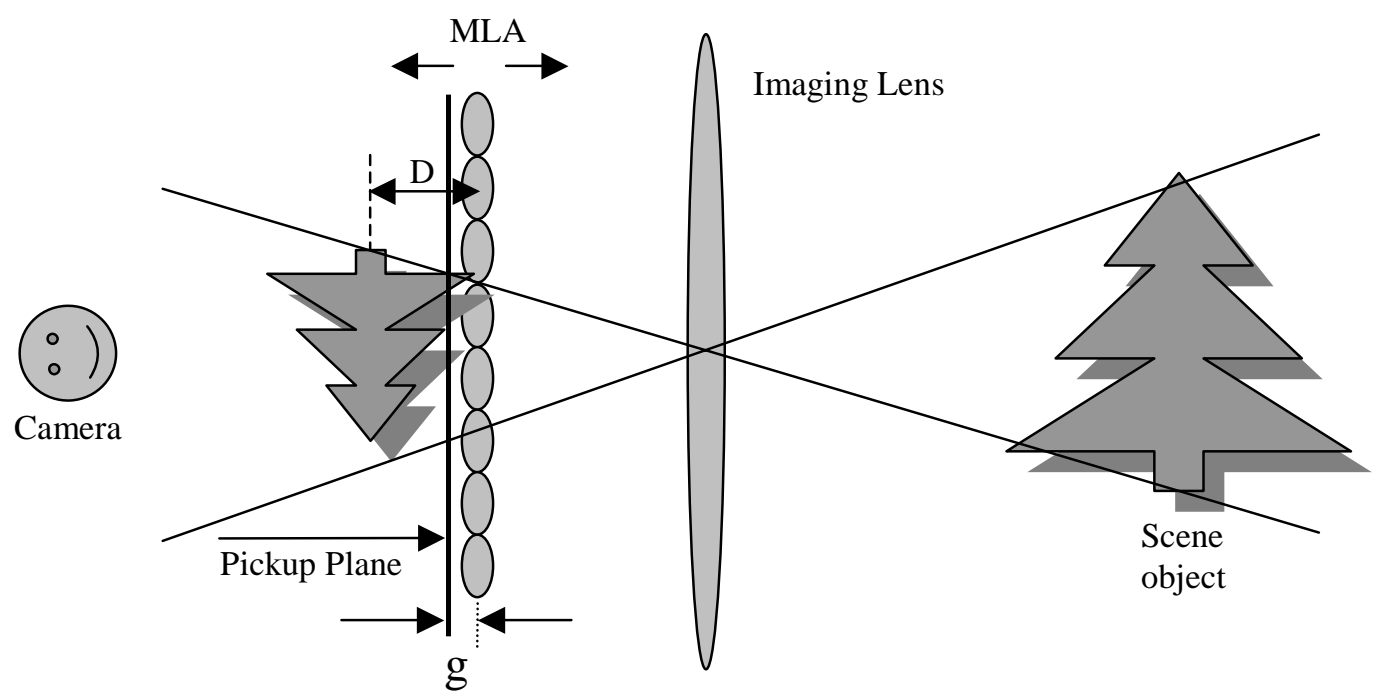

Fig. 1. Single stage IP capturing setup for production of orthoscopic real images (distances not in scale).

\section{MICROLENS FORMATION AND LENS ARRAY SETUP}

The microlens structures typically used in Integral Photography are square or hexagonal-shaped plano convex microlenses which exhibit a higher fill-factor than other structures such as spherically-shaped microlenses.

The optical parameters that are needed for the construction of each microlens of the MLA are the index of refraction $n$ of the microlens material and the focal length $f$ of the microlens. Specifically, the lensmakers formula for bi-convex lenses is given by:

$$
\frac{1}{f}=(n-1)\left(\frac{1}{R_{1}}-\frac{1}{R_{2}}\right)
$$

where $R 1$ and $R 2$ are the radius of curvature of the two convex surfaces respectively. In the case where plano-convex lenses are considered, Equation 1 yields: 


$$
R=(n-1) f
$$

where $R$ is the radius of curvature of the convex surface. Equation 2 relates the radius of curvature $R$ of the convex surface of a plano-convex lens to the optical characteristics $n$ and $f$ as described above.

Utilizing the scripting language of POV-Ray, the microlenses are formed using Constructive Solid Geometry (CSG) techniques. Each microlens is formed as the intersection of a sphere and a parallelepiped or a hexagonal prism in order to produce square or hexagonal lenslets. In either case, the formed microlenses are fully apertured and the radius $R$ of the sphere corresponds to the radius of curvature of the convex surface.

In order to minimize interference problems occurring in adjacent microlenses, a square or hexagonally shaped thin plano-convex lens was realized, by properly adjusting the relative positions of the parallelepiped or the hexagonal prism and the sphere. For square MLAs the displacement $d$ of the parallelepiped from the center of the sphere is given by:

$$
d=\sqrt{(R)^{2}-(p / 2)^{2}}
$$

where $p$ is the pitch of the microlens array. For hexagonal MLAs, the displacement $\mathrm{d}$ of the hexagonal prism from the center of the sphere is given by:

$$
d=\sqrt{(R)^{2}-\left(\frac{p}{2 \cos \left(30^{\circ}\right)}\right)^{2}}
$$

The 3D structure of the square and hexagonal microlenses is shown in Fig. 2 and Fig. 3 respectively.

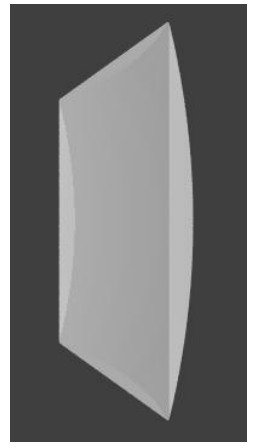

Fig. 2. Simulated square-based microlens in 3D view

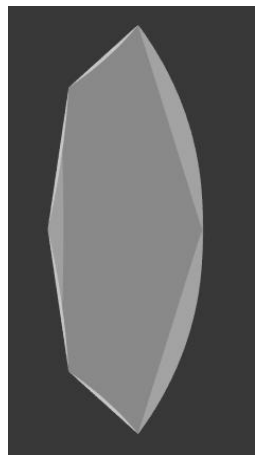

Fig. 3. Simulated hexagonal-based microlens in 3D view

A lens array can be formed as a CSG union of a symmetrical grid of microlenses as depicted in Fig. 4 and Fig. 5 where the elemental images, as seen with the microlens arrays constructed with the above described technique are presented. Lens arrays formed using the above methodology, were tested with the ray-tracer and caused the smaller interference problems due to the minimization of each microlens surface area that is in contact with its closest neighbors.

From its nature, IP is very demanding in display resolution requirements in order to produce high-quality 3D images. Common LCDs have a display resolution ranging from 90dpi to 120dpi, which is not sufficient for the reproduction of photorealistic 3D images. For IP 3D visualizations, high resolution LCDs or hardcopy printing must be used. 


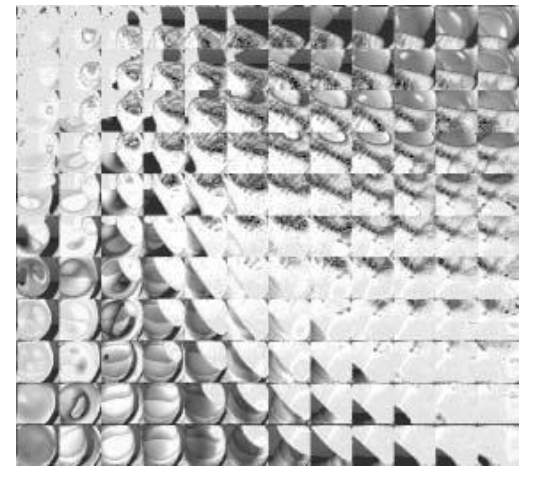

Fig. 4. Square lens array with $1 \mathrm{~mm}$ pitch

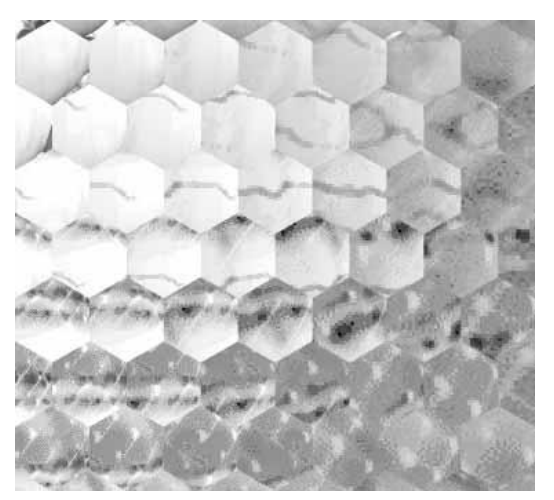

Fig. 5. Hexagonal lens array with $2.3 \mathrm{~mm}$ pitch

The number of microlenses used in the scene is determined based on the display resolution. This number also depends on the ray-tracer output window size. Specifically, for square MLAs, the total number $\mathrm{n}$ of microlenses seen by the camera is given by:

$$
n=\left(25.4 * \frac{w}{d * p}\right)^{2}
$$

where $w$ is the ray-tracer's output image width in pixels, $p$ is the pitch of each microlens in mm and $d$ is the resolution of the display device in dpi. Equation 5 yields a total number of 10404 microlenses when a 600dpi resolution is considered along with a window size of 2400x2400 pixels and an MLA pitch size set to $1 \mathrm{~mm}$.

\section{EXPERIMENTAL RESULTS}

A sample scene in the advanced scene examples of POV-Ray is used as a test scene for the evaluation of the IP capturing system. A fish is in the air above the water surface. The fish skin and eyes are textured with image-maps. Two textured stems are positioned behind the fish at a distance having different depths but close to each other. The fish and stems are reflected in the water below. Three omni-directional lights along with two stems were added in order to increase the complexity of the scene, creating a 3D scene containing a total of five lights and four stems, as depicted in Fig. 6.

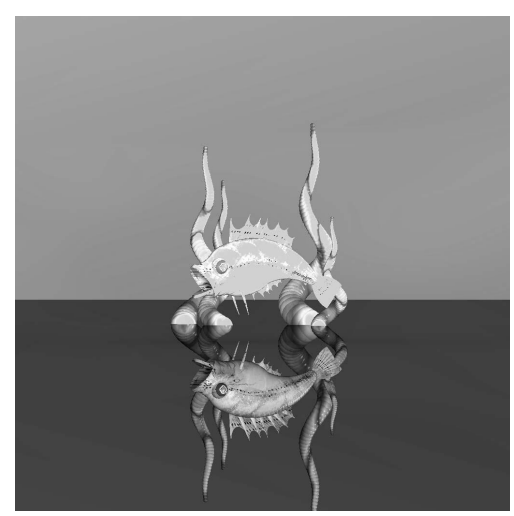

Fig. 6. The test 3D scene 
The ray-tracer's advanced capabilities such as antialising, diffuse and ambient light, texture patterns and computation of reflected, refracted and transmitted rays were used for producing photorealistic results. Moreover, bounding boxes have been utilized to speed up ray-object intersection testing, which is very useful for $3 \mathrm{D}$ scenes with increased complexity.

Our detailed setup is shown in Fig. 7. The imaging lens is a composition of two plano-convex large aperture lenses. The distance of the fish measured from the center of its body to the imaging lens is $100 \mathrm{~cm}$ while the center distance of its image is $41.5 \mathrm{~cm}$ from the imaging lens. The microlens array is placed at the image space, while the camera is placed at a distance of $145 \mathrm{~cm}$ from the imaging lens. The exact camera location is critical to the quality of the IP image, since it is directly related to the number of pixels in each elemental image in the captured integral image (considering a given field of view).

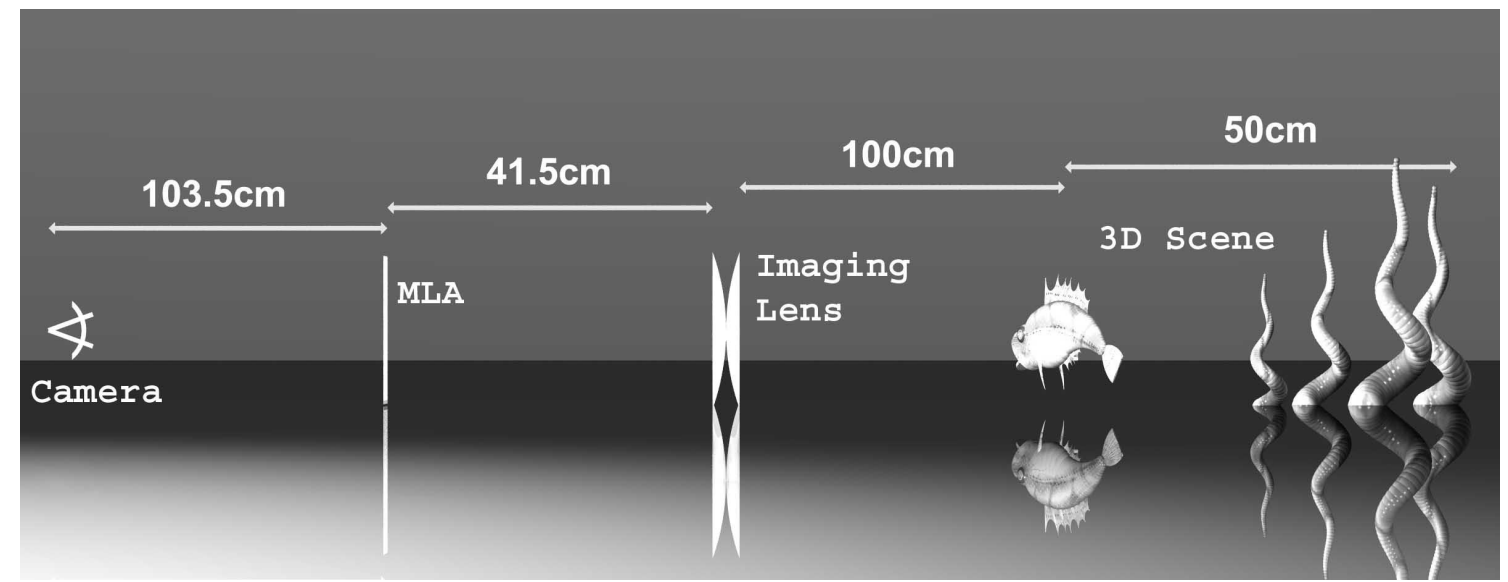

Fig. 7. Single stage IP capturing setup for production of orthoscopic images, real or virtual (distances and object lengths not in scale)

A three-dimensional IP display can be realized combining an appropriate lens array with a high-resolution LCD screen or a hardcopy print. In both cases, the MLA used should match the one modeled in the ray-tracer.

Orthoscopic IP images can be created either in real or in virtual mode depending on the position of the reconstructed 3D image relative to the display plane. Furthermore, the quality of the 3D image depends strongly on the distance from the display plane. A virtual 3D image is formed at a certain depth behind the display panel and exhibits smooth parallax and higher intensity than a real one. On the other hand, a real 3D image is formed in the space between the observer and the display panel thus presenting a more attractive and realistic scene. Therefore, in order to produce a high quality photorealistic 3D image, it is often preferable to combine a real and a virtual 3D image.

In our setup, the production of real or virtual orthoscopic images depends on the exact position of the MLA in the image space. Positioning the MLA at the end of the image space towards the imaging lens, we end up with a pseudoscopic virtual image, which is finally translated to an orthoscopic real image. At the reconstruction stage such an image is formed in front of the display plane. In this case the gap between the lens array and the display device is $g_{r}$ $>f$.

Positioning the MLA at the other end of the image space (towards the camera), a pseudoscopic real image is produced, which is finally translated to an orthoscopic virtual image. At the reconstruction stage such an image is formed behind the display plane. In this case the gap between the lens array and the display device is $\mathrm{g}_{\mathrm{v}}<f$.

The produced IP images were observed at the reconstruction stage with a microlens array of focal length $3.3 \mathrm{~mm}$. For the orthoscopic real images the gap $\mathrm{g}_{\mathrm{r}}$ was $4.8 \mathrm{~mm}$ while for the orthoscopic virtual images the gap $\mathrm{g}_{\mathrm{v}}$ was equal to that of the focal length, due to manufacturing restrictions of the decoding MLA (usually the background substrate has thickness equal to the focal length). The integral images for four successive MLA positions relative to the central plane of the fish body are shown in Figs. 8(a)-(d) in order to demonstrate the transition from real to virtual 3D images. 
The integral images were rendered with a rendering window size of 2400x2400 pixels and resolution of 600 dpi for hardcopy print.

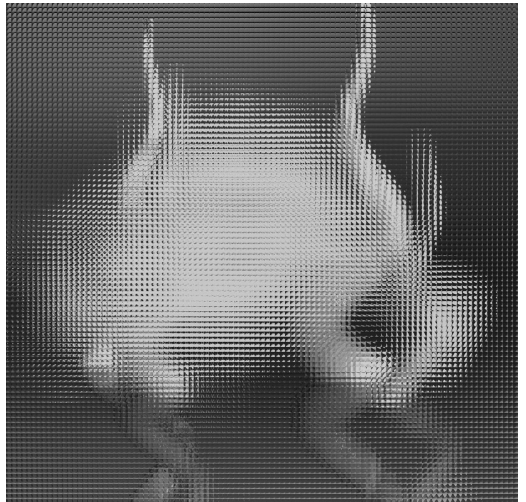

(a)

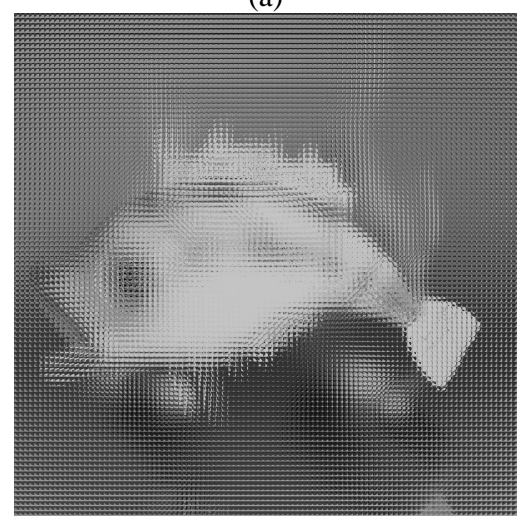

(c)

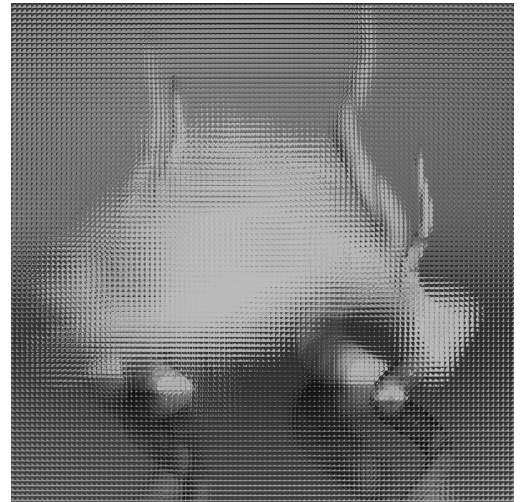

(b)

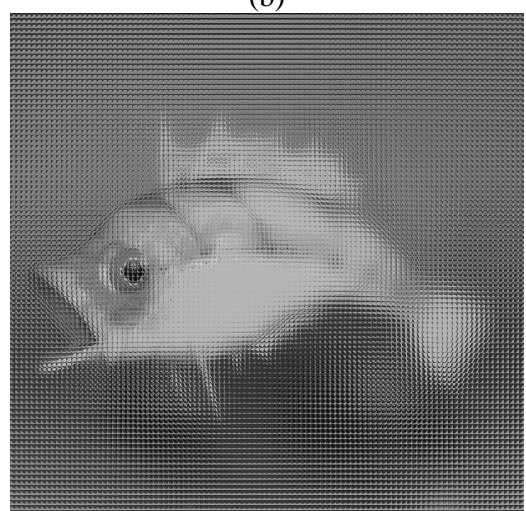

(d)

Fig. 8. (a) MLA $6 \mathrm{~cm}$ towards the imaging lens (the whole scene is located in front of the MLA orthoscopic real image), (b) MLA $4 \mathrm{~cm}$ towards the imaging lens (the fish is located in front of the MLA, while the stems are located just behind the MLA), (c) MLA $2 \mathrm{~cm}$ towards the imaging lens (the fish is located just in front of the MLA, while the stems are behind the MLA), (d) MLA $1 \mathrm{~cm}$ towards the camera (the whole scene is located behind the MLA - orthoscopic virtual image)

\section{BENCHMARKING}

It must be noted that the proposed method produces integral images of high quality and detail. Moreover it takes advantage of most photorealistic effects that can be implemented in modern ray-tracer software. In this way almost every kind of a 3D scene can be rendered as an IP photorealistic image. Nevertheless one key issue in evaluating the method's limitations is the overall performance of the method in time units. Such benchmarks can provide significant input for future extensions by pointing out time-consuming stages of the procedure. The results often determine new pathways as the development of new algorithmic techniques or the implementation of certain procedures in hardware, trying to improve time efficacy of an algorithm.

For the purposes of our work the time needed for each rendering is measured, taking into account a number of factors that directly affect the outcome. Namely the factors considered in our simulation is the Rendered Window Size (RWS) measured in pixels and the Number of Lenses in a Render Window (NLRW), which is inversely proportional to the MLA pitch size and the resolution of the display device. The NRLW is projected on a logarithmic scale axis in order to cover a wide variety of lens array sizes and pitches.

In order to provide reproducible results that are machine invariant at least to a certain extent, a normalized time scale is introduced. The relative time $t_{R}$ acquired in this manner is given by: 


$$
t_{R}=\frac{t_{S R}}{T}
$$

The time needed for each simulation run $t_{S R}$ is divided by a base time unit $T$ which is the time needed to complete a rendering of the original test-scene with its specification attributes summarized in Table 1. By adopting this line of action the complexity of the original scene can be considered as a constant factor for all successive renderings.

It should be noted that in our effort to produce photorealistic results the antialias threshold level is kept at the value mentioned in Table 1. A simplified strategy with no antialising decreases the rendering times by $37 \%$ but produces inferior results. In all renderings five omni-directional lights were used instead of the original two lights, in an attempt to improve the photorealism of our scene. Additionally, successive renderings in which full fill-factor hexagonal geometry MLAs were used, yielded almost identical results (10\% increase in rendering time) with the above used full fill-factor square MLAs. All previous factors remain unaltered in all renderings of the benchmark process.

Table 1. Attribute Specifications for original-test scene rendering

\begin{tabular}{|c|c|c|c|c|c|}
\hline Scene & RWS (in pixels) & Antialias Threshold & No of lights & No of Stems & T (s) \\
\hline Fish13.pov & $720 \times 720$ & 0.3 & 2 (omni) & 2 & 40 \\
\hline
\end{tabular}

Our methodology for evaluating the performance of the whole procedure consisted of multiple renderings that covered different square RWS providing Integral Images targeted to all kind of possible IP applications. Since the lenslet's pitch determines the spatial sampling rate in image pickup, the NLRW is varied by subsequent modifications of the lenslets's pitch size covering MLAs with lenslet pitch in the range of $1-9 \mathrm{~mm}$. The relative time units were extracted by the use of Equation 6 and the results are summarized in Fig. 9.

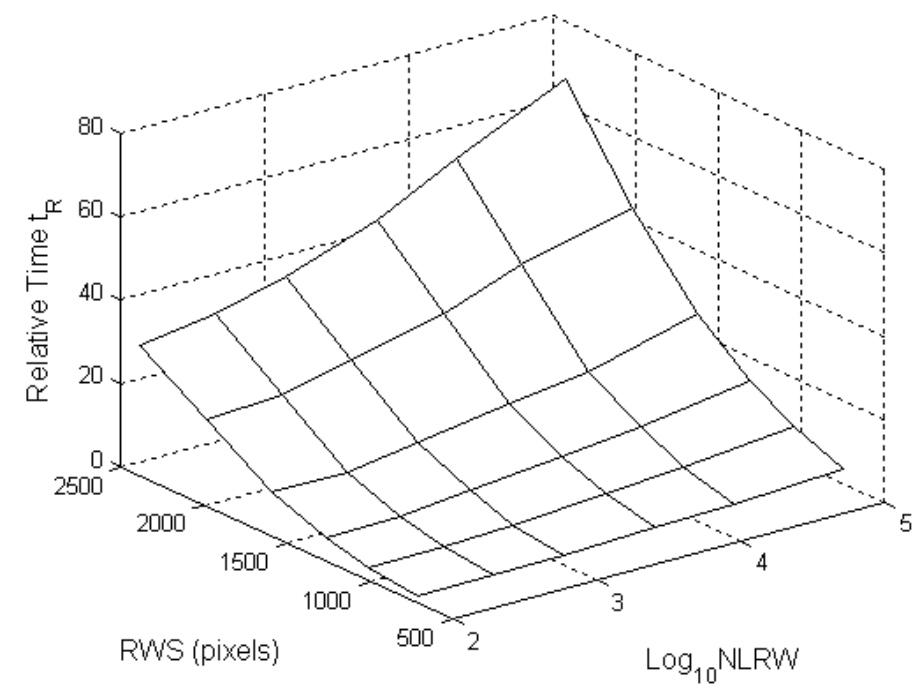

Fig. 9. Benchmark results for simulated Integral Image capturing using square MLAs

One of the main points that should be stressed in the benchmarking procedure is that the complexity introduced by the number of lenses radically affects the time needed to complete a rendering for a specific RWS. When combined with an increase of the RWS, which is supposed to have a predictable but non-linear behavior, increases rendering times significantly.

The time performance evaluation runs were carried out on a typical Intel Pentium IV@3GHz Hyperthreding CPU equipped with 1GB of RAM using an ASUS V9280 (GeForce4 Ti4200) graphic card. 


\section{CONCLUSIONS}

In our work, a novel way for producing high quality, photorealistic Integral Images is proposed, using an advanced general-purpose ray-tracing software package by modeling the necessary optics like ordinary objects of the 3D scene. The modularity of the proposed method allows the modeling of various lens array types and capturing setups. Apparently, these modules can be straightforwardly integrated in a ray-tracer package, while taking advantage of its optimized algorithms and ray-tracing capabilities including texture mapping and other effects.

This procedure offers full control over image depth and positioning of the reconstructed 3D scene. Besides, the modeling of an MLA using real world parameters such as index of refraction and MLA pitch, further ensures that the reconstructed IP scene is of optimal quality. In addition, the introduction of an imaging lens in our setup provides the capability of producing real or virtual images.

Moreover our study provides an excellent tool for further simulation and estimation of novel capturing methodologies implemented in a ray-tracer package that is targeted but not limited to Integral Imaging. Additionally, our technique is evaluated over a wide variety of MLA pitches and rendered window sizes, deriving relative computational times for ease of reproducibility of the results. It is evident that the computational times needed for the simulation of our IP setup, for a given window size, are highly depended on the complexity introduced by the number of lenses contained in a rendered window.

\section{FUTURE WORK}

Based on the above conclusions, more work should be done in modeling optical structures of increased complexity, in order to address various IP capturing methodologies. It is clear that the simulation results are characterized of their remarkable quality, nevertheless, the rendering times are far from considered as real time. Thus, we estimate that augmented ray-tracing techniques should be considered that mainly incorporate hardware acceleration of certain parts of the ray-tracing procedure. Recent technological advances in hardware accelerated graphic cards ${ }^{7}$ along with hardware implementations of ray-tracers on FPGAs ${ }^{8}$ are promising candidates for real time IP generation engines.

\section{ACKNOWLEDGMENTS}

This research was co-funded by $75 \%$ from E.C. and $25 \%$ from the Greek Government under the framework of the Education and Initial Vocational Training Program - Archimedes.

\section{REFERENCES}

1. G. Lippmann, "La Photographie Integrale," Comptes-Rendus Academie des Sciences 146, pp 446-451, 1908.

2. B. Lee, S. -W. Min, S. Jung, and J.-H. Park "A three-dimensional display system based on computergenerated integral photography," J. Soc. 3D Broadcast. Imag. 1, pp 78-82, 2000.

3. Y. Igarashi, H. Murata, M. Ueda "3D Display System Using a Computer Generated Integral Photograph" Japan J.Appl.Phys. 17, pp 1683-1684, 1978.

4. Graham Milnthorpe, Malcolm McCormick, Neil Davies "Computer Modeling of Lens Arrays for Integral Image Rendering," IEEE Computer Society, Proc. of EGUK'02, pp136-141, 2002.

5. Ju-Seog Jang, Bahram Javidi "Formation of orthoscopic three-dimensional real images in direct pickup onestep integral imaging," Optical Engineering 42 No. 7, pp1869-1870, 2003.

6. http://www.povray.org

7. Timothy J. Purcell, Ian Buck, William R. Mark, Pat Hanrahan "Ray Tracing on Programmable Graphics Hardware," ACM Transactions on Graphics 21(3), pp 703-712, 2002.

8. Joshua Fender, Jonathan Rose "A High-Speed Ray Tracing Engine Built on a Field-Programmable System," IEEE International Conf. On Field-Programmable Technology, pp 188-195, 2003. 